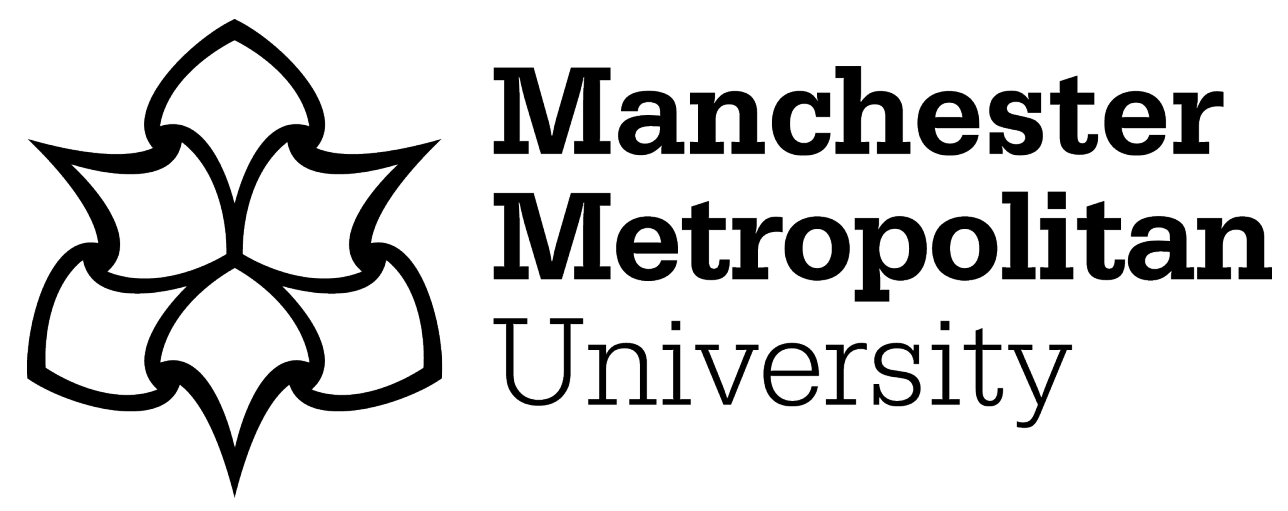

Davies, L, Vagapov, Y, Grout, V, Cunningham, S ORCID logoORCID: https://orcid.org/0000-0002-5348-7700 and Anuchin, A (2021) Review of Air Traffic Management Systems for UAV Integration into Urban Airspace. In: 28th International Workshop on Electric Drives: Improving Reliability of Electric Drives (IWED), 27 January 2021 - 29 January 2021, Moscow, Russia.

Downloaded from: https://e-space.mmu.ac.uk/627424/

Version: Accepted Version

Publisher: IEEE

DOI: https://doi.org/10.1109/IWED52055.2021.9376343

Please cite the published version 


\section{Review of Air Traffic Management Systems for UAV Integration into Urban Airspace}

\author{
Lee Davies \\ Glyndwr University \\ Wrexham, UK \\ Stuart Cunningham \\ Manchester Metropolitan University \\ Manchester, UK
}

\author{
Yuriy Vagapov \\ Glyndwr University \\ Wrexham, UK
}

\author{
Vic Grout \\ Glyndwr University \\ Wrexham, UK
}

\author{
Alecksey Anuchin \\ Moscow Power Engineering Institute \\ Moscow, Russia
}

\begin{abstract}
The role of Unmanned Aircraft Systems have increased substantially in recent years and are now not only used for personal use but for commercial, search and rescue and military application. The increase of the UAS will pose a significant safety risk to not only buildings and property but to the public and general air travel. This increase will undoubtedly cause a significant strain on Air Traffic Control (ATC) system and will lead to UAS not being used to their full potential. The use of autonomous UAS will increase over the coming years, and a reliable system of Unmanned Traffic Management (UTM) will be needed both for effective safety and reliability. Currently, there is no real framework in place to accommodate low level UAS in urban airspace. This research aims to discover the current state of the art technologies and innovations developed to create a workable UTM framework giving an overview of the various methods available to analyse the likelihood of a UTM being developed. The findings of the paper show that there is a definitive need for such a system to be developed and maintained if UAVs are to be incorporated into everyday life.
\end{abstract} UTM

Keywords-UAS, drones, UAV, sense and avoid, ATC, ATM,

\section{INTRODUCTION}

Over the last decade, the use and development of UAS have increased drastically as the monetary benefits of producing drones are realised. The role of the humble Unmanned Aircraft Vehicle (UAV) has far outgrown its beginnings and is now used in a multitude of different disciplines. These include power line inspection, railway inspection, utility pipes inspection, telecommunication transmission and inspection, coast guard surveillance and traffic surveillance to name but a few of the UAV versatile uses. This list is almost endless due to the adaptability if the current crop of UAS. Currently, in the United Kingdom, there are restrictions in place in order to ensure that drones are UAVs must not be operated within $50 \mathrm{~m}$ of people, buildings, cars, boats and trains. An operator must also ensure that the UAV is at least $150 \mathrm{~m}$ away from groups of over 1,000 people [1]-[3]. This itself presents a significant problem for UAV operators to fly in low level airspace, especially when attempting to fly over towns and cities. Airports are another high level danger to consider as recently the rules for operating a drone around an airfield has changed especially after the Gatwick and Heathrow incidents involving drones. Currently, it is illegal to fly in a restricted airfield zone at any time unless with express permission by the ATC [4]-[6].

UAS have for outgrown its humble beginnings and are now seen as an essential tool in everyday life. The exceptional growth of the utilisation of drones has seen them being used in various fields, and the projected GDP uplift in these sectors is extremely encouraging. With a substantial increase in economic increase, reaching into billions (Fig. 1). However, with the need for UAS in lower class airspace, there must be a robust and easily adaptable system in place for both piloted and autonomous aircraft to be safely controlled in the skies. The goal of achieving such a system is not without its challenges as there will be a level of automation required. The need for a system is desirable in the UK, and there must be an appropriate level of organisation and clarity, and it must be based around a regulatory framework [7]. With the scale of UAV densities increasing in the skies, accommodating new users for both commercial and hobbyists with existing users is going to be a challenge (Fig. 2). In terms of safety, the hobbyists who may well be less experienced than commercial pilots will be operating their UAV's in close proximity which will also lead to significant challenges.

Currently, in the UK BVLOS operations are only permitted with Civil Aviation authority approval [8],[9]. However, stride has been taken to support BVLOS operation within the UK, and this will be an important step forward when it comes to UTM systems. The CAA has seen that BVLOS is an important factor in the evolution of UAVs and UAS and has published literature detailing the approach to drone deliveries and inspections [10].

Change is needed and that the current model of ATC and UTM is not adequate to support the arrival of UAV's in their current forms. Adaption and evolution is needed in order to fully realise the potential that UAVs have.

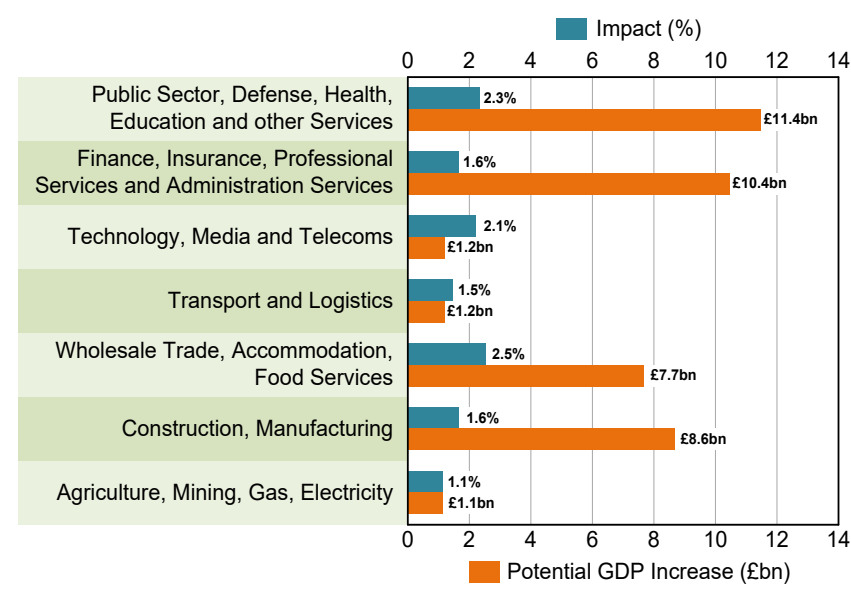

Fig. 1. Projected GDP Uplift of drones in the UK by 2030; data from [11]. 


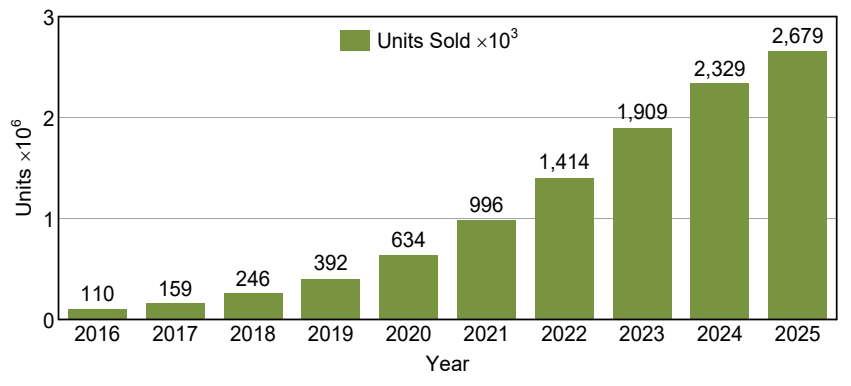

Fig. 2. Projected growth sales; data from [12].

\begin{tabular}{|c|c|c|}
\hline \multicolumn{3}{|c|}{ UAV potential applications to urban areas and smart cities } \\
\hline $\begin{array}{l}\text { Traffic monitoring and } \\
\text { management }\end{array}$ & $\begin{array}{c}\text { Green urban areas } \\
\text { management and monitoring }\end{array}$ & Merchandise delivery \\
\hline $\begin{array}{l}\text { Health emergency } \\
\text { services }\end{array}$ & Tourism support & UAV taxi \\
\hline $\begin{array}{l}\text { Security and crowd } \\
\text { monitoring }\end{array}$ & UAV-based surveying & $\begin{array}{l}\text { UAV-aided wireless } \\
\text { communications }\end{array}$ \\
\hline $\begin{array}{l}\text { UAV-based infrastructure } \\
\text { inspection }\end{array}$ & Disaster management & $\begin{array}{l}\text { Data collection in wireless } \\
\text { sensor network }\end{array}$ \\
\hline
\end{tabular}

Fig. 3. UAV applications to urban areas and smart cities [13].

This paper focuses on the review of the current state of the art technologies that have been developed or theorised to provide a robust and adaptable UTM framework that is reliable. The paper looks at various iterations of design ranging from complex algorithms to Concept of Operations (CONOPS) in order to focus on the enhanced functional abilities of ATM for UAS applications. The technologies have been investigated in terms of efficiency, reliability and adaptability.

\section{RISK ASSESSMENT}

In order for there to be a workable and flexible model that can be utilised to safely have UAS inhabiting the skies, there has to be a comprehensive risk assessment and management protocols in place. Using basic risk assessment criteria is the first step forward in achieving the basics of safely operating a UAV in a congested area.

With the roles of UAVs changing rapidly, the projected growth of commercial drones' sales is forecasted to grow significantly since 2016. It is fair to assume that these figures could increase even further than what is predicted such is the versatility and functionality of UAVs.

\section{A. Classification of UK Airspace}

The Civil Aviation Authority (CAA) has divided the UK airspace into five classes, these being, A, C, D, E and G and defines the flight rules that must be applied to provide the minimum air traffic services. The classes of A, C, D and $E$ are designated as controlled airspace, whereas $G$ is defined as uncontrolled airspace.

Class $\mathbf{G}$ allows aircraft to fly whenever and wherever they choose but are governed by a set of simplistic rules. There are no legal requirements for pilots to notify ATC as it is the pilots who are solely responsible for their own and other users' safety.

\section{B. Risk Assessment Safety Grid}

Using a rating grid, it is possible to identify hazards and place them in accordance to the level of acceptable risk (Fig. 4). These being:

\begin{tabular}{|c|c|c|c|c|c|}
\hline Severity & Minimal & Minor & Major & Hazardous & Catastrophic \\
\hline Frequent & LOW & MEDIUM & HIGH & HIGH & HIGH \\
\hline Probable & LOW & MEDIUM & $\mathrm{HIGH}$ & $\mathrm{HIGH}$ & HIGH \\
\hline Remote & LOW & MEDIUM & MEDIUM & HIGH & HIGH \\
\hline $\begin{array}{l}\text { Extremely } \\
\text { Remote }\end{array}$ & LOW & LOW & MEDIUM & MEDIUM & HIGH \\
\hline $\begin{array}{l}\text { Extremely } \\
\text { Improbable }\end{array}$ & LOW & LOW & LOW & MEDIUM & MEDIUI \\
\hline
\end{tabular}

Fig. 4. Safety risk assessment grid [14].

- High (Unacceptable Risk) - Must be mitigated to a medium or low risk prior to implementation.

- Medium (Acceptable Risk - Operations may be implemented but extra safety requirements are introduced to increase the safety margin.

- Low (Acceptable Risk) - May be implemented with safety requirements

\section{UTM CONCEPT OF OPERATIONS APPROACH}

There has been an influx of work undertaken in this field as many researchers have understood the benefits of having an operational ATC and UTM system specifically designed for low level UAS.

In 2016 Kopardekar et al. [15] published a paper detailing the concept of operations (CONOPS) for a UTM system. Taking a logical viewpoint, the researchers realised that the system would need to be flexible in certain areas but would also need to be rigid and controlled within other areas. One of the standout features of the system is that the model could be operated without any human interaction to monitor vehicles. The model that Kopardekar at al. suggest is aimed at the low risk situations in order to generate the initial data. However, any developed model will evolve into a more complex and higher risk factored situations and environments.

Working closely with NASA, the paper presents two initial categories of UTM systems for safe drone operation. One is a portable model that can be transported and implemented in areas that require support for operations. The second is a mode which has constant availability to a determined geographical area. Both measures could ensure that BVLOS operations are completed effectively and more importantly, safely.

The system is designed to be used for Class $G$ airspace by offering an appropriate level of transparency so any BVLOS mission can be tracked and identified from an ATC. The design of the UTM uses a combination of operation procedures, an array of automation systems, integrated vehicle capabilities, airspace design and a combination of flight rules. The first phase of the design will allow UAV's to be flown and permitted in areas where the probability of a collision with other users would be a rare occurrence, and this process would require a small degree of infrastructure. The next phase would have UAV operations increased to allow the significant increase of air traffic, which would result in more services and infrastructure being implemented. The last phase would see the UTM model accessible to all future scenarios. These procedures are hoped to aid the integration of UAVs into 
the airspace as they expand in numbers over the coming years.

Another method of developing a UTM CONOPS system was presented by Johnson et al. [16]. The system uses a risk-based planning strategy as the centre of its CONOPS and relies on 'big data' and the Internet Of Things to initiate a robust trajectory planning design. The system uses a combination of data regarding ground-based structures and population masses in view along with debris modelling in a bid to calculate the percentage of probable risk that is usually associated with UAV operations. The objective of the design is to successfully identify the trajectories that will reduce the risk of collision when operations are undertaken and to weigh up and strike a workable balance between the needs of the UAV operators in terms of being granted access to densely populated areas whilst identifying and reducing the risk to livestock, buildings and people. The aim is to fully integrate UAVs with conventional traffic so that they can operate effectively and safely.

Entitled the Glasgow UTM Development Process, the design sets out the process in which the systems tools are presented so that UAVs are permitted to operate autonomously in high populated areas. Using contemporary census data, the researchers produced a model associated to population densities that would be within the experiments proposed corridor. They were able to use the data alongside a simulation of predicted traffic within the area of the proposed corridor to run a calculation based on the level of risk is far better detail than the recommended practices set out by the International Civil Aviation Organisation (ICAO). The designed simulation is designed to allow the additions of new abilities so that it can calculate the level of risk at certain locations within the proposed framework model.

The design is still in the relatively early stages and is set to evolve as the test results thus far have been positive. The model itself has shown that there is a definite increase in both usability and visualisation and can be utilised to produce very detailed technical data along with providing detailed predictive outcomes for an event. The model also provides support for new trajectory planning and scheduling with the added aid of predicted calculations of risk level based on the UAV's trajectories that are fed into the model. This will allow for the improvement of UAV trajectory planning when used in line with the multiple classificational criteria based in SARPs [17].

In 2016 Jiang et al. [18] proposed a CONOPS and system architecture for UAS traffic management. The system lends ideas from large scale ATC protocols with inherent several key differences to provide UAS with a way of being able to manoeuvre in safe airspace. The system takes in to account all UAV's fundamental differences in terms of manoeuvrability, range and function. They also pose the notion of establishing a web based interface for all pilots to have the option of submitting approved flight plans and have the ability to access relative and crucial data to plan operations. The paper details who has the authority of UTM and it also looks how to develop a physical architecture which would be used to build a large scale UTM.

The UTM system requires that it has its own designated airspace with its own manger(s). This is styled upon the existing Terminal Radar Approach Control (TRACON) which operates with areas having assigned airspace and ATCs.

Taking in to account the Federal Aviation Administration's (FAA) strong role regarding the regulations surrounding UAV's, the design respects that the FAA would need to continue as a point of authority over all UAV regulations and safety. The system itself would require at least one or two controllers or managers to oversee the operations on a daily basis; this is to ensure that all pilot using the corridor adhered to the strict protocols that would be in place. The managers would also be responsible for liaising with the public, law applications, emergency service and to ensure that all UTM computerised services are working correctly.

Having crucial data available in a web based digital format is vital for the smooth running of the design, and this would consist of GPS, regional weather stations, appropriate agencies and ground-based radar.

One of the problems facing the overall design is that it insists that all the UAVs must be equipped with sense and avoid (SAA) capabilities in order to prevent a rare catastrophic case of the automated control fail. ADS-B has been viewed as a reliable method of SAA technology as it is a high accuracy satellite based surveillance system applicable for reliable determination of UAV's airspeed and relative location. The airspeed and location data would be recoded and transmitted to the ground stations formed into a network.

The UTM is proposed to be categorised into four classes: (1) Pilots; (2) Control Centres; (3) UAS; (4) Airspace Infrastructure. Within the categories, subsystems will be added to support the UTM system. Also, the paper suggests that flight authorisation, whilst still being regulated by the FAA should be streamlined so that the process can be speeded up and become more efficient. One suggestion is to exclude UTM managers form the flight plans reviewing procedure. This would only work if there were rules in place which allowed for operation outside UTM systems much in the same way aircraft fly over certain airspace areas where no support and control of radar systems. The suggestion is that rural and less populated areas can be controlled by a UTM system which is less advanced comparing to the areas that it is required in.

Drawing from the conclusions, it is inevitable that once a workable UTM has been implemented, then it is only natural that a more complex and reliable system will be developed due mainly to the fact that the technology will only improve. The paper sees the research as a first step of the realisation of the CONOPS and is an aspirational one at best with the hope that one can be implemented in the next five years, this is something of which will undoubtedly not happen due to the current legislation and technology available. The paper does address that there is still much work to be done on the system as there are still areas of UAS traffic management that requires more comprehensive research.

However, this proposal is encouraging as it relies heavily on both advanced technology and human interaction with keeping many of the crucial decisions with the UTM operators and not relying on AI or a specific algorithm. Out of the all the papers that have produced a CONOPS the paper presented by Jiang et al. [18] is one of the concepts more likely to been implemented sooner rather 
than later as it does not rely too heavily on advanced technology.

\section{UTM IN Cities}

Following on from his previous work, Lundberg et al. [19] produced research in the potential side effects of the implementation of a UTM in cities. The paper details the sampled side effects of the implementation produced by simple UTM system controlling autonomous point to point drone traffic. It describes the use of interactive simulations which consisted of synthetic images and appropriate statistic data so that the simulated scenarios could be developed. The premise was to identify key issues with UTM systems. A Detect and Avoid (DAA) system was used to manage potential conflicts between the drones and consisted of two layers. Firstly, a global DAA system and secondly a local and distributed DAA based on the drones. Again, the UTM operator is responsible for the managing of the traffic load as well as overseeing that the system is operating correctly.

The researchers ran the simulation by using a case study. The city of Norrköping was used due to the fact that the city has low buildings but also has an airport close to the city centre. The simulation consisted of a drone simulator which was programmed to execute a real-time simulation of drone traffic in a particular airspace area. The system uses two varieties of design as guidance for the drones. The first is defined by the division of flight altitude into layers and the second method is a conflict avoidance algorithm. However, each drone is still subject to single or several outer regulations. The researchers also developed a UTM toolkit so that they were able to operate drone traffic in the proposed simulator effectively. Using a Dijkstra heuristic to guide the traffic, they hoped to navigate the drones from start to finish whilst avoiding the geofenced that were put in place.

Using the simulation, the researchers were able to detect and record the side effects of a UTM system. The system found that with the design relying on two levels of DAA would provide the local DAA operation to prevent system activation for conflicts between registered drones. This means that this would be used for obstacles in the vicinity and unregistered drones. The global DAA should be used to measure the distance around and below the drones. Using geofencing is also important to the overall design as this helps all traffic to automatically re-route around these areas. The researchers also introduced a third level of intervention. They deemed that layering was capable of increasing airspace capacity by means of vertical separation.

From these first point interventions, the researcher found that they had a direct and undesired effect on their samples. They found that the global DAA system was attempting to force traffic off the planned route and into the geofencing that had been set up. Another side effect was the perceived slow reaction or con-compliance of drones that strayed into geofenced areas. They saw that the drones were unable to adjust their trajectory to avoid entering a geofenced area. This alerted the researchers to the notion that the UTM systems need a method of prediction, detection and prevention in this area.

Using a city, the size Norrköping they were presented with significant problems as the airport is within the city and they were forced to present geofencing around the airport in relation to the landing and take-off areas. From this, they observed due to the implementation of the geofencing that flight zones of the drones were increased and thusly the routes were increased. Continuing with the use of geofencing, the researchers used different layers to address the congestion when geofencing is used with high levels of traffic. From the simulation, they found that most of the traffic flowed in two directions which devalued the layered approach. However, they found that using altitude separation can reduce head-on conflicts; however, they were unable to control the speeds of the drones, which would impact on the increase of potential collisions.

Furthermore, the researcher used a direct point to point traffic solution. Simply, the system would have the ability to reserve the air corridor mainly on basis: first come, first serve.

The study attempted to address the management of low level airspace operations. To attempt to rectify the side effects, it is evident that a more advanced design of dynamic airspace components is needed. The paper suggests that UTM in a city can be developed if it focuses on management of urban airspace. This does not come without implications such as side effects when using the UTM tools, management of the new airspace components and managing unexpected issues.

To promote a safe and workable system in order to provide ease of access to different users, the design needs to have an established robust and flexible way of managing and planning within the desired airspace. The further development of this UTM approach is discussed in [20], [21].

\section{Low Altitude UTM CONCEPT}

Labib et al. [22] produced a concept model for a low altitude airspace multilayer model for UTM. The aim of the work is to primarily address the problems associated with UTM systems by suggesting a model which can be used in in low altitude uncontrolled airspace. This work itself discusses the weighted multilayer network comprising the nodes and air paths as an investigation into how the structure and the relevant capacity of UAV traffic allowed for safe operations.

To achieve the results needed, a series of test simulations based around configurations of UAV traffic densities were conducted to validate the proposed model. Because of nature of complexity of analysing network systems, the researchers envisage a more general multilayer network framework. Each of the layers represents different paths that have differentiating properties embedded into them such as velocity and traffic capacity.

For the UTM algorithm design itself, the researchers lead to a version of a distributed design where the UAVs within the designated airspace can plan their routes using locally generated data. The decision making procedure is supported by an ad-hoc communication system. It is hoped that it would be the model to be scaled over a greater distance. It was decided that the algorithm would not just focus on the optimisation of distances and time, but it would also focus on the optimisation of travel time whilst considering the limitations of energy efficiency. The researchers drew inspiration from their previous works on aerial vehicle management in using an inverted ant colony optimisation approach and the exploration of a stable connectivity algorithm used for UAV swarms for wide area 
monitoring. The design consists of a model in which the area of class $\mathrm{G}$ airspace remaining uncontrolled is divided into horizontal layers with their own altitude. Then, each layer presented as a node in a scheme where airpath with the nodes being able to be assigned to multiple layers.

The optimisation of the pathways is critical for the effectiveness of the model and proposed a formula for achieving this. The aim was to efficiently reduce the total time of travelling and the overall energy consumption associated with the UAVs operation within the travelling grid. Each layer has a capped allowance for the number of traffic in each airway so that safety is not compromised.

For the experimentation process, the research categorised three heuristics to approach the problem. The first category comprises of a path planning method. The second category delivers a probability approach to design the model based on the assumption of up to date global knowledge and the network traffic conditions. The third category was described as a pheromone guided greedy approach which utilises the data of the local air traffic conditions. The UAVs aim to follow a pre-determined path which has been calculated to be the shortest route from the take zone to the destination, but if they encounter congestion in the airway, each UAV within the network then computes a probabilistic decision calculation applies on one of the two pre-determined responses. Either to hover in a queue within the layer or to take an alternative shortest route based on using the same algorithm that was used to originally calculate the shortest path. For the third phase, the UAVs rely on the data of the current traffic conditions and the UAVs proceed by following the calculated path until the amount of traffic on the next airway becomes higher than a pre-defined threshold level. It is at this stage in the operation where the UAV will deploy a pheromone trail which acts as a deterrent to other close proximity UAVs which alters the UAVs that there is a problem and that an alternative airway should be sought.

The simulation evaluated the reliability of the design. The results of the experiment demonstrated that the performance alters drastically, and it was observed that the UAVs local decision making ultimately lead to a decrease in the number of traffic on the more widely used areas which improved the performance in traffic at both a global and local level. They also found that there were significant improvements for larger traffic samples compared to the smaller traffic samples. The simulation also detailed the UAVs decision making in which they saw that the UAVs tended to change between layers which give the indication that the UAVs decision making to avoid congested areas led to an improvement of the global system in terms of overall traffic distribution.

Researchers clarify that although promising, any future work on the system must be based on focusing on a more realistic scenario whilst changing the operating limits and exploring more realistic communication methods.

\section{UTM Mission Planning Optimisation Simulation}

Another approach was adopted by Tan et al. [22] in which described an ambitious concept through a simulation. The proposed design was based around optimising mission planning for UTM scenarios by designing a route planning method for multiple UAV operations. The system comprises of a pathfinding algorithm along with a designated scheduling system to ensure the safe use of the given airspace. The pathfinding is achieved by generating points from a heuristic search and by calculating the shortest distance between the take-off and the destination points. Secondly, permitted flights will be scheduled accordingly to avoid the possibility of conflicts and are done through an Evolutionary Algorithm (EA). The embedded system is responsible for the flow of traffic by acting accordingly to delay flight requests or to delay them if the algorithm deems that its unsafe for the UAV to take flight at that given time. This is done to reduce both congestions and the risk of conflicts in the airspace and is enabled by the route planning and scheduling being known in advance to take-off.

This framework consists of three main components. Firstly, the construction of the traffic network, which presented as a combination of links between nodes and the potential aerial hazards defined as constraints. The network must be able to function at a safe distance from buildings and is split into multiple layers of airlocks as well as endeavouring to have as much connectivity as safely possible.

The second component is the route planning algorithm which is able to handle a multitude of flight requests and generate suitable pathways. The algorithm used was split into two separate modes for the users. These are categorised as the intersection mode, where the paths have overlaps, and the non-intersection mode has no overlaps to decrease the probability of aerial conflicts.

The final aspect is the scheduling of the flights. The system requires that the user or operator submits a flight plan with the take-off location, take-off time, maximum delay estimation and destination before any flight can commence. With this information, the algorithm can generate flight paths for users which may have overlaps. To allay the probability of conflict, time scheduling is proposed and is based on the assumption that all UAVs will travel at a constant speed.

The experimentation was conducted through a simulation of Singapore's local town airspace where takeoff and destination sites were selected separately in commercial, residential and industrial categories. Flight requests were generated randomly through a 900 -second cycle and EA algorithm would then calculate the time delay needed and the probability of collisions of the flights that had been submitted. Within this, each UAVs flight request is examined carefully and the feasibility of completion calculated and if the time separation is within the parameters the flight path deemed with the highest probability of conflict with be rejected until the conflict probability has been reduced to no conflict.

To identify any problems within the design, a delivery mission was initiated by planning routes that of delivery zones that ranged from the three categorised urban areas. Once the parameters are in place, the simulation was started using a range of 10-200 flight requests comprising a start point, landing zone, take-off time. EA then calculated whether to accept, reject or delay each flight respectively.

Each planned route that was produced to minimise the total distance and their fore flight time congestion is likely to occur as the route planning system was deemed to be greedy in nature. This ended up creating the probability of congestion in certain areas. It was seen that the smaller the area resulted in a higher percentage of rejections. This is related to the limitation of airspace available at that time. 
The system itself was capable to determine routes with shortest distance based on flight operation data, and it was also able to schedule flights according to prevent the possibility of collisions. The researches deem that the EA is a viable tool for the route planning system and is able to adaptive for urban airspace management. From the results, they found that the constraints of the perceived risk in the simulation should be more constrained to add other factors to the airspace. These include other airborne objects and other UAV users, and that other factors other than distance need to be considered carefully in the pathfinding method, for example, population avoidance, energy capacity and efficiency.

\section{CONCLUSION}

With ever increasing designs and novel applications of UAVs, there is always going to be an increase in the number of unmanned systems in low level airspace. For the future of drones to be realised especially in the roles of delivery and courier services, a safe and manageable UTM system is not just needed, it's a necessity. It is evident that the realisation of such a system is still some way off and that no workable framework has been devised even through the best efforts. The papers present a sound basis for the development of a UTM system using different approaches, and all show that it is not a simple problem that can be easily overcome. There are many working variables that must be overcome. The fact that SAA systems are still in their infancy and are an integral part of a UTM system proves that there is still much work to do to find a solution. The simulations themselves show a positive step forward but are not set in a real world scenario, as weather, the rashness of other air users and acts of god are not included in the design process. From the research undertaken, much of the work is heavily based on a level of autonomy which presents its own challenges of problems. Having a combination of autonomy and human control is the most reasonable step forward as the role of human intuition and reaction cannot be underestimated. The research presents solid work in the field and will obviously expand over time as the state of the art technology increases, but for now, there is very little chance of a UTM system being implemented anytime soon to accommodate the increase of UAVs. It is evident that UAVs will become part of low level airspace and that their adaptability will increase but that based on future work.

\section{REFERENCES}

[1] "Introduction - Dronesafe." Dronesafe. https://dronesafe.uk/ (Accessed Nov. 08, 2020).

[2] R. Khan, S. Tausif, and A.J. Malik, "Consumer acceptance of delivery drones in urban areas," International Journal of Consumer Studies, vol. 43, no. 1, pp. 87-101, Jan. 2019.

[3] B. Rahmadya, R. Sun, S. Takeda, K. Kagoshima, and M. Umehira, "A framework to determine secure distances for either drones or robots based inventory management systems," IEEE Access, vol. 8, pp. 170153-170161, 2020.

[4] “Airfield restrictions - Dronesafe.” Dronesafe. https://dronesafe.uk/ restrictions/ (Accessed Nov. 08, 2020).

[5] L. Qingdong, Y. Jiaquan, L. Fei, and L. Xin, "Airfield clearance zone design theory and realization based on Gauss mid-latitude algorithm," in Proc. 5th IEEE International Conference on Computer and Communications (ICCC), Chengdu, China, 6-9 Dec. 2019, pp. 183-187.

[6] S.V. Shvetsova, and A.V. Shvetsov, "Ensuring safety and security in employing drones at airports," Journal of Transportation Security, online first, 2020, doi: 10.1007/s12198-020-00225-z
[7] "Drones - regulatory framework background." EASA. https:// www.easa.europa.eu/domains/civil-drones-rpas/drones-regulatoryframework-background (Accessed Nov. 08, 2020).

[8] Beyond Visual Line of Sight in Non-Segregated Airspace: Fundamental Principles and Terminology, 2nd ed. UK CAA, 2020. [Online]. Available: https://publicapps.caa.co.uk/docs $/ 33 / \mathrm{CAP} \%$ 201861\%20-\%20BVLOS\%20Fundamentals\%20v2.pdf. Accessed: Nov. 19, 2020.

[9] L. Davies, R. C. Bolam, Y. Vagapov, and A. Anuchin, "Review of unmanned aircraft system technologies to enable beyond visual line of sight (BVLOS) operations," in Proc. 10th International Conference on Electrical Power Drive Systems (ICEPDS), Novocherkassk, Russia, 3-6 Oct. 2018, pp. 1-6.

[10] "Setting the path to drone deliveries and remote inspections: Making beyond visual line of sight drone operations commonplace." UK CAA. https://www.caa.co.uk/News/Setting-the-path-to-dronedeliveries-and-remote-inspections--Making-beyond-visual-line-ofsight-drone-operations-commonplace/ (Accessed Nov. 19, 2020).

[11] "Drones could add £42bn to UK GDP by 2030 - PwC research." PwC. https://www.pwc.co.uk/who-we-are/regional-sites/northernireland/press-releases/drones-0518.html (Accessed Dec. 08, 2020)

[12] K. Buchholz. "Commercial drones are taking off." statista.com https://www.statista.com/chart/17201/commecial-drones-projectedgrowth (Accessed Dec. 08, 2020)

[13] N. Mohameda, J. Al-Jaroodi, I. Jawhar, A. Idries, and F. Mohammed, "Unmanned aerial vehicles applications in future smart cities," Technological Forecasting and Social Change, vol. 153, Art. no. $119293,2020$.

[14] L.C. Barr, R.L. Newman, E. Ancel, C.M. Belcastro, J.V. Foster, J.K. Evans, and D.H. Klyde, "Preliminary risk assessment for small unmanned aircraft systems," in Proc. 17th AIAA Aviation Technology, Integration, and Operations Conference, Denver, USA, 5-9 June 2017, pp. 1-57.

[15] P. Kopardekar, J. Rios, T. Prevot, M. Johnson, J. Jung, and J.E. Robinson III, "Unmanned aircraft system traffic management (UTM) concept of operations," in Proc. 16th AIAA Aviation Technology, Integration, and Operations Conference, Washington, DC, USA, 1317 June 2016, pp. 1-16.

[16] C. Johnson, and M. McKay, "Interactive risk-based planning for high -density unmanned airborne system ('drone') traffic management (UTM)," in Proc. 35th International System Safety Conference, Albuquerque, USA, 21-25 Aug. 2017, pp. 1-9.

[17] "SARPs - Standards and recommended practices." IKAO. https:// www.icao.int/safety/safetymanagement/pages/sarps.aspx (Accessed Nov. 19, 2020)

[18] T. Jiang, J. Geller, D. Ni, and J. Collura, "Unmanned aircraft system traffic management: Concept of operation and system architecture," International Journal of Transportation Science and Technology, vol. 5, no. 3, pp. 123-135, 2016.

[19] J. Lundberg, K. Palmerius, and B. Josefsson, "Urban air traffic management (UTM) implementation in cities - sampled sideeffects," in Proc. 37th IEEE/AIAA Digital Avionics Systems Conference, London, UK, 23-27 Sept. 2018, pp. 1-7.

[20] J. Lundberg, M. Arvola, C. Westin, S. Holmlid, M. Nordvall, and B. Josefsson, "Cognitive work analysis in the conceptual design of firstof-a-kind systems - designing urban air traffic management," Behaviour and Information Technology, vol. 37, no. 9, pp. 904-925, 2018

[21] J. Lundberg, C. Westin, M. Arvola, S. Holmlid, and B. Josefsson, "Cognitive work analysis and conceptual designing for unmanned air traffic management in cities," in Proc. European Conf. on Cognitive Ergonomics, Utrecht, The Netherlands, Sept. 2018, pp. 1-4.

[22] N. Labib, G. Danoy, J. Musial, M. Brust, and P. Bouvry, "A multilayer low-altitude airspace model for UAV traffic management," in Proc. 9th ACM Symposium on Design and Analysis of Intelligent Vehicular Networks and Applications, Miami Beach, FL, USA, 28-29 Nov. 2019, pp. 57-63.

[23] Q. Tan, Z. Wang, Y. Ong, and K. Low, "Evolutionary optimizationbased mission planning for UAS traffic management (UTM)," in Proc. International Conference on Unmanned Aircraft Systems, Atlanta, GA, USA, 11-14 June 2019, pp. 952-958. 